\title{
Transiciones familiares y trayectorias laborales femeninas en el México urbano*
}

\author{
Orlandina de Oliveira* \\ Marina Ariza**
}

\begin{abstract}
Resumo
A partir del recuento de la serie de transformaciones estructurales que explican la creciente participación femenina en la actividad económica, se explora la diversidad de trayectorias laborales de un conjunto de mujeres urbanas, casadas y con hijos, nacidas entre 1940 y 1970, con base en los relatos contenidos en sus historias de vida. Se analiza en particular la influencia de dos transiciones familiares, casamiento y nacimiento de los hijos, en el grado relativo de discontinuidad laboral. El análisis sugiere que el peso de estas transiciones es diferencial por sector social, y se expresa entre otras cosas en una distinta duración en la interrupción de la trayectoria laboral. En la interpretación de la heterogeneidad de las trayectorias se recurre tanto a factores socioculturales, como demográficos y económicos.
\end{abstract}

Palabras-chave: Actividad Económica Femenina, Transformaciones Estructurales, Transiciones Familiares, Trayectorias Laborales, Relaciones de Género.

\footnotetext{
* Trabajo presentado en la 94th Annual Meeting de la American Sociological Association, en la Special Session: Gender, Careers, and the Life Course in Comparative Perspective, agosto de 1999, y publicado en GoMES, Cristina. (comp.) Procesos sociales, población y familia. Alternativas teóricas y empíricas en las investigaciones sobre vida doméstica. México, Flacso y Editorial Porrúa, 2001. Recebido para publicação em novembro de 2001.

* Profesora-investigadora de El Colegio de México.

** Investigadora del Instituto de Investigaciones Sociales de la Universidad Nacional Autónoma de México.
} 
Trayectorias laborares femeninas

Family Transitions and Female Work Trajectories

in Urban Mexico

\begin{abstract}
After reviewing main structural transformations leading to increased female economic activity, the paper examines the diversity of female work trajectories in a selected group of urban married women with children, born between 1940 and 1970. Analysis is based on their life histories and takes as its object of inquiry the influence of two central family transitions (marriage and birth of children) in the relative discontinuity of female work trajectories. Discussion suggests a differential influence of such transitions in the discontinuity of work trajectories by social sector, observed in the duration of work interruptions. Sociocultural, demographic and economic factors are used to explain such differences.
\end{abstract}

Key words: Female Economic Activity, Structural Transformations, Family Transitions, Work Trajectories, Gender Relations. 
Orlandina de Oliveira, Marina Ariza

\section{Introducción}

En el contexto internacional, la sociedad mexicana se ubica en un rango medio en lo que se refiere a los niveles de participación económica de la población femenina, con valores promedios en 1997 alrededor del $37 \%$ en la población femenina de 12 años y más. Aun cuando se trata de un grado de participación relativamente bajo en comparación con el prevaleciente en los países de Europa Occidental y Norteamérica, el mismo se ha alcanzado gracias a un acelerado proceso de crecimiento de la actividad económica femenina que ha incorporado a decenas de miles de mujeres mexicanas al mercado de trabajo en las últimas dos décadas.

Esta creciente inclusión en la actividad económica extradoméstica no hubiera sido posible sin una serie de transformaciones sociales de diversa índole que enmarcan su ocurrencia. El descenso de la fecundidad y la elevación de la escolaridad han jugado sin duda un papel sobresaliente, pero también la terciarización y los recientes procesos de crisis y reestructuración económica por las que ha atravesado la región. $\mathrm{Si}$ bien la carrera laboral ininterrumpida está lejos de ser la trayectoria dominante de las mujeres mexicanas de fin de siglo, la entrada a la actividad laboral es una transición cada vez más frecuente en el conjunto de las que integran sus cursos de vida. Perseguimos en este trabajo recoger la heterogeneidad de trayectorias laborales de un grupo de mujeres urbanas pertenecientes a dos cohortes de nacimiento (1940-1955 y 19561970), evaluando la colisión relativa entre los eventos del mundo familiar y el laboral a través de cortes transversales realizados en distintos momentos de sus itinerarios de vida. Para ello dividimos el artículo en dos partes: en la primera se hace un recuento de las principales tendencias estructurales de largo plazo que han contribuido al incremento de la participación económica femenina, describiendo las distintas modalidades que asume esta 
Trayectorias laborares femeninas

participación; en el segundo se abordan directamente - con base en el análisis de entrevistas en profundidad - los tipos de trayectorias encontradas según el grado de discontinuidad de la vida laboral

\section{El contexto económico y familiar: las transformaciones de largo plazo}

Muchas son las transformaciones sociales que anteceden a la creciente vinculación de las mujeres con la actividad económica extra-doméstica. Acontecen tanto en el ámbito demográfico, como en el económico, el cultural y el socio-institucional, por citar sólo algunos de los más evidentes. Situados en el plano económico y tomando un horizonte más o menos amplio, sobresale el proceso de terciarización como la tendencia secular más claramente conectada con la cada vez más importante presencia femenina en los mercados de trabajo. ${ }^{1}$ Ya desde los tempranos inicios de la centuria, allá por la década de 1920, este sector de la economía mostró con claridad su marcada preferencia por el empleo femenino; ${ }^{2}$ tendencia que se iría acentuando conforme la terciarización se abría paso como la transformación de largo plazo en que desembocaría la mayoría de los cambios económicos por

\footnotetext{
${ }^{1}$ La creciente terciarización de la fuerza de trabajo constituye una tendencia en curso en los países desarrollados desde el siglo pasado. En la actualidad más del $70 \%$ de la fuerza de trabajo de un conjunto de países desarrollados (Canadá, USA, Europa nórdica) se concentra en ese sector de actividad. A diferencia de éstos, los países en desarrollo se caracterizan por un sector terciario de menor tamaño, menos diversificado y con predominio de los servicios sociales, distributivos y personales. De acuerdo con los estándares internacionales, la economía mexicana ha pasado a ser desde mediados de los 90 una economía terciaria, puesto que en el sector se aloja más del $50 \%$ de la fuerza de trabajo.

2 OliveirA, Orlandina de; ArizA, Marina, y ETERnod, Marcela. La fuerza de trabajo en México: un siglo de cambios. En LEÓN, José Gómez de, y ROMERO, Cecilia A. Rabell. Cien años de cambio demográfico en México. México, FCE, 1999 [en prensa].
} 
transcurrir. Terciarización y feminización ${ }^{3}$ del mercado de trabajo fueron así las dos caras de un dilatado proceso de cambio socioeconómico que ampliaría considerablemente las oportunidades de inserción económica de las mujeres ${ }^{4}$, coincidente con otros no menos importantes como la urbanización y las grandes transferencias de población del campo a la ciudad.

Esta ampliación de los espacios laborales disponibles para la población femenina propiciada por la terciarización mostró diferentes ritmos e intensidades, según el momento histórico y los rasgos estructurales de la economía. Así, en los años de auge del llamado crecimiento estabilizador (1930-70), la expansión del empleo tuvo lugar preferentemente en los subsectores modernos del terciario (servicios sociales y al productor); mientras que en las décadas finiseculares de crisis y reestructuración productiva (19701995), fueron los subsectores menos ventajosos en términos relativos (el comercio $y$, en menor medida, los servicios personales) los que mayor cabida dieron a la creciente incursión femenina en el mundo del trabajo. Este cambio en la naturaleza del proceso de terciarización hacia la expansión de los sectores no modernos de los servicios, ha ido aparejado del fortalecimiento de las formas no asalariadas de inserción (trabajo por cuenta propia y ayuda familiar no remunerada), aspecto que profundiza la de por sí considerable heterogeneidad del sector. ${ }^{5}$

3 La acentuada feminización del terciario se manifiesta en el elevado número de mujeres, 79 en 1995, por cada 100 hombres; proporción muy superior a la presencia femenina en el conjunto de la fuerza de trabajo. ID., IB.

4 Se estima que más de la mitad del crecimiento de los sectores no manuales ocurrido entre 1960-90 en América Latina obedece al empleo femenino. ARRIAGADA, Irma. La participación desigual de la mujer en el mundo del trabajo. Revista de CEPAL, n ${ }^{\circ}$ 40, 1990, pp.87-104.

${ }^{5}$ Investigaciones realizadas para mediados de los 90 señalan que el trabajo por cuenta propia y la ayuda familiar no remunerada se expanden con ritmos superiores al trabajo asalariado; lo mismo puede decirse del crecimiento del comercio en relación con los subsectores modernos del terciario (servicios sociales y al productor). El crecimiento del trabajo por cuenta propia y del no remunerado ha sido mayor en la fracción femenina de la fuerza de trabajo: entre 
Trayectorias laborares femeninas

La permanencia de la división sexual del trabajo como eje de organización social es otro de los factores que decididamente ha influido sobre la actividad (doméstica y extra-doméstica) de las mujeres en distintos momentos históricos. Cada una de las diversas estrategias económicas implementadas en el país desde mediados de la centuria ha supuesto a su vez una particular vinculación entre la familia y el mercado, un modo de organización sexual del trabajo, con un impacto variable sobre la constitución de los sectores femeninos de la fuerza de trabajo. Así, a diferencia de lo que aconteció durante los años de vigencia del modelo agro-exportador (1895-1930), y mucho después, en el período de crisis y reestructuración económica (1970-1995), la estrategia de industrialización por sustitución de importaciones (1930-1970) produjo una relativa masculinización del mercado de trabajo al desestimular la inserción de las mujeres en la actividad económica extra-doméstica. Procesos tales como la modernización de la producción artesanal (ocurrida entre 1910 y 1930), la conformación de una mano de obra asalariada, la capitalización de la agricultura y el crecimiento de las industrias de bienes de capital y consumo duradero, se sustentaban en un modelo de división sexual del trabajo que escindía las esferas de la reproducción y producción económica en mundos prácticamente opuestos, replegando a las mujeres al primero de éstos.

Esta relativa polarización genérica ocurría en un entorno social caracterizado por un alto crecimiento demográfico, con fuertes presiones sobre las funciones reproductivas de la mujer y elevadas tasas de fecundidad (de aproximadamente 7 hijos por

1970 y 1995 su porcentaje en la PEA femenina pasó de 26.6 a 40.5\%. GARCÍA, Brígida. Fuerza de trabajo en 1995. Las implicaciones del nuevo modelo de desarrollo. México, Demos/IIS-UNAM, 1996; Economic Restructuring, Women Survival and Transformation in Mexico. Trabajo presentado en el Seminario Female Empowerment and Demographic Processes, Suecia, abril de 1997; GARCía, Brígida y OlIVEIRA, Orlandina de. La participación femenina en los mercados de trabajo. Trabajo, año 1, no 1, 1998, pp.139-161. OliveirA, O.; ARIzA, M. y ETERNOD, M. La fuerza de trabajo en México: un siglo de cambios. Op. cit. 
mujer), tornando difícil para ellas la armonización entre los mundos del trabajo y la familia. El tipo de organización familiar predominante en esos años (mayormente nuclear y de familia numerosa), es el que más se asemeja en términos ideales al modelo del jefe proveedor exclusivo. Los niveles de vida de la población y la expansión económica sostenida que por varios años el modelo de sustitución de importaciones logró producir, convertían en realidad la existencia de un salario familiar suficiente para la supervivencia familiar. Otros aspectos de índole ideológica o pública contribuían a la marcada orientación familística predominante en esos años, como la exaltación desde el Estado de los valores familiares y de la domesticidad. ${ }^{6}$

En virtud de la división sexual del trabajo antes referida y de los condicionantes familiares y contextuales que pesaban sobre las mujeres, son las jóvenes y solteras las que muestran la mayor disposición relativa a participar en la actividad económica en este período histórico (1930-1970). Así lo confirma la caída generalizada en las tasas de actividad económica a partir de los 25 años en los distintos grupos de mujeres trabajadoras. Parece muy plausible, por tanto, que para la mayoría de las mujeres de esa época el trabajo conservara un valor estratégico o coyuntural, como una actividad suplementaria que se realiza en circunstancias muy precisas, siempre que las necesidades familiares así lo requieran. En consecuencia, en sentido general el grado de compromiso con la actividad económica extra-doméstica en la

${ }^{6}$ En los años 50, por ejemplo, el Estado mexicano premiaba la fecundidad de las mujeres prolíficas. AlBA, Francisco. La población en México: evolución y dilemas. México, El Colegio de México, 1977; LeÑERo OtERO, Luis. Estudios sobre el avance de las políticas de población en México 1980-1988. Revisión y validación de las actividades en población sus alcances y obstáculos. México, El Colegio de México, 1989; SANDOVAl ARRIAGA, Alfonso. Veinte años de política de población en México: Elementos para una recapitulación crítica. Mimeo, 1993; ARIZA, Marina. Migración, familia y participación económica. Mujeres migrantes en una ciudad caribeña. En LATTES, A.; SANTiBáñez, J. y CAstillo, M. A. (coords.) Migración y Fronteras. México, El Colegio de la Frontera Norte/Asociación Latinoamericana de Sociología/El Colegio de México, 1998. 
Trayectorias laborares femeninas

población femenina en estos momentos es relativamente bajo, si bien han de esperarse importantes diferencias por clases y sectores sociales.

Un panorama algo distinto se vislumbra en los años más recientes de crisis y reestructuración económica (1970-95). En éstos existen aspectos de la demanda y la oferta laboral que flexibilizan relativamente el esquema anterior de organización sexual del trabajo, sin modificar en realidad sus rasgos básicos. Por un lado, muchas de las nuevas inversiones realizadas en el período manifiestan una demanda específica de mano de obra femenina. Al mismo tiempo, los cambios promovidos en las condiciones laborales hacia una mayor flexibilidad y competitividad de cara al mercado mundial $\mathrm{y}$ el establecimiento de prácticas de subcontratación, estimulan la inserción de sectores de mujeres a los que las tales condiciones (trabajo a tiempo parcial, por cuenta propia y a domicilio) permiten "compatibilizar" mejor sus roles productivos y reproductivos. ${ }^{7}$ En el mismo sentido, la ampliación del terciario, que en este período se expande por encima de la PEA industrial, ofrece oportunidades crecientes de inserción a las mujeres, principalmente en el sector comercio. ${ }^{8}$ No obstante, estas oportunidades surgen en su gran mayoría en los sectores más precarios del terciario, donde los ingresos por hora son más bajos y las condiciones de seguridad laboral más inciertas. ${ }^{9}$

\footnotetext{
7 Este aspecto ha llevado a afirmar a los especialistas que la oferta de trabajo flexible es una característica de la participación económica femenina. CRUZ PIÑEIRO, Rodolfo. Volatilidad en el empleo femenino: características individuales y del hogar. Frontera Norte, vol. 6, n⿳ 12, julio-agosto 1994; CERRUTTI, Marcela. Coping with Opposing Pressures: A Comparative Analysis of Women's Intermittent Participation in the Labour Force in Buenos Aires and Mexico City. Tesis de doctorado, Texas, Universidad de Texas en Austin, 1997.

8 Se estima que entre 1991 y 1995 el comercio creció a una tasa anual de 6.6 frente al 3.7\% de los otros servicios. (ENE, 1991 y 1995.)

9 Oliveira, O.; ArizA, M. y ETERNOD, M. La fuerza de trabajo en México: un siglo de cambios. Op. cit.
} 
Desde el punto de vista de la oferta laboral se han modificado algunas de las condiciones que inhibían una participación más activa de las mujeres en el mercado de trabajo. El descenso acelerado de la fecundidad ha sido sin duda un cambio de la mayor trascendencia. En 1970 la tasa global era de aproximadamente 7 hijos por mujer, en 1997 se estimaba en 2,65. Es importante acotar que el impacto de la caída en la fecundidad sobre el aumento de la participación económica ha sido diferencial por grupos de edad y generaciones. Según lo refiere Mier y Terán, fueron las mujeres mayores (35 años y más, nacidas entre 1947 y 1952) las que primero incrementaron su participación relativa en el mercado de trabajo, seguidas secuencialmente por aquéllas situadas en los tramos anteriores de edad (30-34 y 25-29) y nacidas en quinquenios subsiguientes (1952-57). ${ }^{10} \mathrm{El}$ resultado fue que a medida que las generaciones eran más jóvenes aumentaba la frecuencia de la participación económica; mientras en una misma generación la tendencia a participar crecía con la edad. Es por ello que se producen superposiciones en el cambio de los patrones reproductivos y el aumento de la participación económica entre mujeres de diferentes cohortes. ${ }^{11}$

De igual relevancia ha sido el aumento de la escolaridad: más de la mitad de las mujeres de 15 a 29 años ha logrado concluir sus estudios secundarios en un contexto de marcada desigualdad social. ${ }^{12}$ Un papel no despreciable han jugado las

\footnotetext{
${ }^{10}$ La explicación de estos diferenciales reside en el hecho de que el descenso de la fecundidad, acaecido principalmente por la difusión de prácticas anticonceptivas, ha tenido más bien como finalidad reducir el tamaño de la familia, sin modificar la edad a la unión ni los intervalos intergenésicos de primer orden. MIER Y TERÁN, Marta. Implicaciones del descenso de la fecundidad en la participación laboral femenina: el caso de México. Trabajo presentado en el seminario Fertility Transition in Latin America, International Union for the Scientific Study of Population (IUSSP), Buenos Aires, abril de 1990.

${ }^{11}$ ID., IB.

${ }^{12}$ LÓPEZ, Ma. de la Paz. Las mujeres protagonistas de los cambios. Demos. Carta demográfica de México, n 11, 1998, pp.17-18.
} 
Trayectorias laborares femeninas

reiteradas crisis económicas por las que ha atravesado el país en los años recientes. El deterioro de las condiciones de vida y la caída ininterrumpida del salario real, han obligado a los hogares mexicanos a hacer un uso más intensivo y racional de la fuerza de trabajo disponible, incluyendo a las mujeres. ${ }^{13}$

Otros aspectos de índole estrictamente demográfica han contribuido al incremento de la actividad económica femenina. El descenso de la fecundidad y la reducción en el tamaño promedio de los hogares ${ }^{14}$, han modificado la duración de los roles tradicionales de madre y esposa en sentidos contrapuestos: se ha acortado el tiempo dedicado a la reproducción y crianza de los hijos menores de cinco años, mientras se ha extendido la permanencia de la vida en pareja. ${ }^{15}$

En virtud de los procesos mencionados, la participación económica femenina se ha duplicado en el curso de los últimos

\footnotetext{
${ }^{13}$ Entre 1984 y 1996, por ejemplo, la proporción de hogares mexicanos con un sólo perceptor de ingresos se redujo de $58.2 \%$ a $45.8 \%$; la contracción fue aún más severa en los hogares cuyo el jefe recibe menos de dos salarios mínimos mensuales: de $57.4 \%$ a $40.7 \%$. OliveIRA, Orlandina de. Políticas económicas, arreglos familiares y perceptores de ingresos. Demos. Carta demográfica de México, no 12, 1999, [en prensa]; CoRTÉs, Fernando y RuBALCAVA, Rosa María. Autoexplotación forzada y equidad por empobrecimiento: la distribución del ingreso familiar en México, 1977-1984. Jornadas 120, México, Centro de Estudios Sociológicos, El Colegio de México, 1991; RubalCaVA, Rosa Ma. Necesidades, recursos y posibilidades: el ingreso de los hogares mexicanos en el periodo 1984-1994. Tesis de doctorado, CIESAS/Universidad de Guadalajara, 1998.

${ }^{14}$ Entre 1970 y 1995, el tamaño promedio de los hogares se colocó por debajo de los cinco miembros. LóPEZ, M. Las mujeres protagonistas de los cambios. Op. cit.

${ }^{15}$ Como lo refiere López, y otros autores, en 1970 las mujeres con hijos menores de cinco años empleaban 20 años de sus vidas en la reproducción y crianza de sus hijos, tiempo que se ha reducido en aproximadamente 8 años para las que inician su reproducción mediados los 90 . En las condiciones demográficas actuales, un matrimonio mexicano puede aspirar a vivir 40 años en pareja. TUIRÁN, Rodolfo. Demographic Change and Family and Non-Family Related Life Course in Contemporary Mexico. Tesis de doctorado, Texas, Universidad de Texas en Austin, 1998; CONSEJO NACIONAL DE POBLACIÓN - CONAPO. Encuesta Nacional de Planificación Familiar. México, Conapo, 1995.
} 
veinticinco años (1970-1995). ${ }^{16}$ En este incremento ha jugado un papel significativo la actividad económica de las mujeres casadas y con hijos, las que parecen no estar dispuestas a clausurar definitivamente la incursión en el mundo del trabajo. ${ }^{17} \mathrm{~A}$ diferencia de lo ocurrido en el período anterior, ya no se verifica una caída drástica de la participación al llegar a los tramos centrales de la vida reproductiva. En la hipótesis de algunos autores, sin embargo, la creciente participación de las mujeres casadas y con hijos se da predominantemente de manera intermitente. ${ }^{18}$

Un aspecto que denota la menor polarización relativa entre el mundo del trabajo y la familia en los años de crisis y reestructuración económica (1970-1995) en contraste con el período anterior, es el progresivo acortamiento de la distancia entre los niveles de participación económica femenina y masculina. Entre 1970 y 1995 se registraron descensos de al menos 18 puntos porcentuales en las diferencias entre las tasas de actividad de uno y otro sexo. Al entrar al último lustro del siglo, la participación económica femenina se aproxima a la mitad del nivel de la masculina. ${ }^{19}$

\footnotetext{
${ }^{16}$ La tasa de participación era en 1970 de 17.6\%, y en 1995 de 35\%. LÓPEZ, Ma. de la Paz. Las mujeres protagonistas de los cambios. Op. cit.; INSTITUTO NACIONAL DE Estadística, Geografía E INFORMática (INEGI)/SECRETARÍA DEL TRABAJO Y PREVISIÓn Social (STPS). Los hogares en México, México, INEGi/STPS, 1997.

${ }^{17}$ PedReRo Nieto, Mercedes y RENDÓN, Teresa. El trabajo de la mujer en México en los setentas. En SECRETARÍA DE PROGRAMACIÓN Y PRESUPUESTO (SPP). Estudios sobre la mujer 1. El empleo y la mujer. Bases teóricas, metodológicas y evidencia empírica. México, SPP, 1982; GARCíA, Brígida y OLIVEIRA, Orlandina de. Trabajo femenino y vida familiar en México. México, El Colegio de México, 1994.

${ }^{18}$ CRUZ PIÑEIRO, R. Volatilidad en el empleo femenino... Op. cit.

${ }^{19}$ La disparidad en los respectivos niveles de participación puede tomarse como indicador del grado de inequidad en el acceso al trabajo extra-doméstico. Al respecto, los países de mayor equidad relativa en el contexto europeo son Suecia y Finlandia, en los que la brecha en el grupo de los 24 a los 54 años no excede los 7 puntos. Los países mediterráneos (España e Italia), e Irlanda, son los de mayor disparidad, con una diferencia de 40 y 60 puntos. [ORGANISATION FOR
} 
Trayectorias laborares femeninas

No obstante la menor incompatibilidad relativa entre el mundo familiar y el laboral, y la creciente propensión a trabajar de las mujeres, es necesario señalar que esta inclusión ha tenido lugar de manera limitada y segregada, conservando rasgos de inequidad que menoscaban los recursos y potencialidades que el trabajo es capaz de brindar. En este sentido es importante resaltar el efecto contradictorio de los procesos de reestructuración económica sobre las condiciones de las mujeres trabajadoras: abren oportunidades de trabajo pero en condiciones de relativa exclusión. ${ }^{20}$ Estudios recientes muestran que las trabajadoras mexicanas se concentran en actividades precarias (principalmente trabajo por cuenta propia) y/o de tiempo parcial. ${ }^{21} \mathrm{El}$ carácter precario del trabajo por cuenta propia en el caso de las mujeres ${ }^{22}$ queda de manifiesto en la mayor frecuencia con que ellas se

ECONOMiC Co-OPERATION AND DEVElOPMENT (OECD) (ed.) Women and Structural Change. New Perspectives. Paris, OECD, 1994.] México se ubica en un rango próximo a estos últimos, con una disimilitud promedio de 54.4 puntos porcentuales en el valor de la diferencia entre las tasas de participación de hombres y mujeres en el mismo intervalo de edad. [OlIVEIRA, O.; ARIZA, M. y ETERNOD, M. La fuerza de trabajo en México: un siglo de cambios. Op. cit.]

${ }^{20}$ LI, Peter S. y CuRRIE, Dawn. Gender Differences in Work Interruptions as Unequal Effects of Marriage and Childrearing: Findings from a Canadian National Survey. Journal of Comparative Family Studies, vol. 23, Summer, 1992; OliveIRA, Orlandina de y ARIZA Marina. División sexual del trabajo y exclusión social. Revista Latinoamericana del Trabajo, año 3, $\mathrm{n}^{\circ}$ 5, 1997; OlIVEIRA, Orlandina de y ARIZA, Marina. Género, trabajo y exclusión social. Mimeo, 1999.

${ }^{21}$ OliveIRA, O. y ARIZA, M. Género, trabajo y exclusión social. Op. cit.; GARCíA, B. Fuerza de trabajo en 1995. Op. cit.; GARCíA, B. Fuerza de trabajo en 1995. Op. cit.; e Economic Restructuring, Women Survival... Op.cit.

${ }^{22}$ En vista de que por la naturaleza de la actividad, el trabajador por cuenta propia carece de contrato laboral, de prestaciones y de sueldo fijo, su trabajo suele ser catalogado como precario en relación con el asalariado. En estricto sentido, cuando se aplica al estudio de las actividades por cuenta propia, el concepto de precariedad alude a las formas de organización de la actividad productiva o de prestación de servicios por parte del trabajador mismo. Los indicadores refieren casi siempre al tamaño de la empresa, las horas trabajadas y el tipo de ingreso. 
encuentran en empresas unipersonales $y$ en labores de subsistencia (o que reditúan menos de dos salarios mínimos mensuales), trabajando menos de 15 o más de 48 horas promedio semanales. Por su parte, el trabajo de tiempo parcial sobresale por su alto grado de feminización: 107 y 85 mujeres asalariadas y cuenta propia, por cada 100 hombres, realizaban actividades de tiempo parcial en el año en México. ${ }^{23}$ Son conocidas las implicaciones negativas de este tipo de trabajo sobre las trayectorias laborales femeninas, en la medida en que frenan o invierten el posible curso ascendente de éstas. ${ }^{24} \mathrm{De}$ hecho, como lo afirman Li y Currie, gran parte de la desigualdad por género en el ingreso, ya sea en un punto en el tiempo o a través de la vida de las mujeres, puede ser atribuida a la tendencia a trabajar a tiempo parcial o de manera intermitente. Se cuestiona así que el incremento del trabajo de tiempo parcial pueda ser el producto de cuotas crecientes de autonomía femenina; parece más bien una de las pocas alternativas existentes ante la permanencia de una desigual distribución del trabajo doméstico. ${ }^{25}$

${ }^{23}$ Oliveira, O.; ARIZA, M. y ETERnOD, M. La fuerza de trabajo en México: un siglo de cambios. Op. cit.

${ }^{24}$ En contraste con los hombres, la probabilidad de pasar de un trabajo de tiempo parcial a otro de tiempo completo no se incrementa con el tiempo. LI, P. S. y CURRIE, D. Gender Differences in Work Interruptions... Op. cit.; BosCH, Gerhard; DAWkings, Peter y MiCHON, Francois. (eds.) Times are Changing: Working Time in 14 Industrialized Countries. Geneva, Suiza, International Institute for Labour Studies, 1994; OECD. (ed.) Women and Structural Change... Op. cit.; CERRUTTI, M. Coping with Opposing Pressures... Op. cit.

${ }^{25}$ El trabajo de tiempo parcial encierra una amplia variedad de situaciones en cuanto al nivel de calificación, el carácter voluntario (elegido) o involuntario (impuesto por las condiciones del mercado de trabajo), y la duración (relativamente más corto o más largo); factor éste que incide a su vez sobre la posibilidad de obtener protección laboral. La experiencia europea ha mostrado que el desempeño del tiempo parcial suele ser diferencial por sexo. [OECD. (ed.) Women and Structural Change... Op. cit.; Bosch, G.; DAWkINGS, P. y MiCHON, F. (eds.) Times are Changing... Op. cit.] En la población masculina de esos países se limita con frecuencia a los momentos extremos de la vida activa (juventud y vejez), y adquiere el matiz de evento transicional. En las mujeres, en cambio, el 
Trayectorias laborares femeninas

Pero el dato que de manera más contundente expresa las condiciones de inequidad que caracterizan a la inserción económica de las mujeres mexicanas en nuestros días es el referente a la segregación ocupacional: alrededor de un $60.6 \%$ y $41.7 \%$ de los trabajadores por cuenta propia y asalariados, respectivamente, y un $50 \%$ de los de tiempo completo, tendrían que ser redistribuidos para lograr una conformación equitativa de la estructura ocupacional entre los hombres y las mujeres trabajadoras. ${ }^{26}$ Desde nuestro punto de vista y del de otros autores, parte de esta segregación y de las condiciones generales de inequidad por género en el mercado de trabajo son posibles gracias a la permanencia de una desigual distribución entre el trabajo doméstico y extra-doméstico, un modo de división sexual del trabajo que condiciona y limita las posibilidades de inserción económica femenina. En otras palabras, las mujeres están pagando un precio más alto que los hombres por la edificación de la vida familiar. ${ }^{27}$

\section{La diversidad de trayectorias laborales femeninas}

Hasta ahora nos hemos referido a tendencias de cambio de los mercados de trabajo elaboradas a partir de la comparación de

\footnotetext{
trabajo de tiempo parcial es una experiencia típicamente asociada con los años más intensos de la vida reproductiva, con las limitaciones que esta esfera impone a la disponibilidad laboral. [LI, P. S. y CuRRIE, D. Gender Differences in Work Interruptions... Op. cit.; CeRRUTTI, M. Coping with Opposing Pressures... Op. cit.]

${ }^{26}$ OliveIrA, O. y ARIZA, M. Género, trabajo y exclusión social. Op. cit.

${ }^{27}$ A mediados de los 90 , sólo $37.8 \%$ de los hombres mexicanos mayores de 12 años realizaba alguna actividad doméstica, en contraste con el $92.3 \%$ de las mujeres; discrepancia que se acentúa en el curso del ciclo de vida, alcanzando valores máximos entre las personas unidas o casadas con pocos años de escolaridad. ARIZA, Marina y OliveIRA, Orlandina de. Género y clase como ejes de inequidad: una mirada metodológica. Trabajo presentado en el $1^{\circ}$ Congreso Nacional de Ciencias Sociales, Consejo Mexicano de Ciencias Sociales (Comecso), México, D.F., abril de 1999; LI, P. S. y CuRRIE, D. Gender Differences in Work Interruptions... Op. cit.
} 
datos transversales en diferentes momentos del tiempo. La revisión de estudios longitudinales pone de manifiesto que todavía persiste en México una elevada proporción de mujeres que no ha trabajado a lo largo de sus vidas o lo ha hecho en forma esporádica a pesar de la creciente participación económica femenina ocurrida en las últimas décadas. La mayoría de las trayectorias laborales femeninas se caracteriza por su carácter discontinuo, aunque han habido cambios en el país hacia una mayor permanencia de las mujeres jóvenes en actividad laboral después del casamiento o del nacimiento de los hijos. ${ }^{28}$ Análisis para las áreas metropolitanas muestran la mayor inestabilidad o intermitencia de las trayectorias laborales de las mujeres en comparación con los varones, y de las mujeres casadas frente a las solteras. ${ }^{29}$ Para la Ciudad de México se ha visto que los más altos niveles de escolaridad se asocian con una mayor permanencia de las mujeres en la fuerza de trabajo, y que las esposas, con o sin hijos pequeños, presentan una menor propensión a permanecer en la fuerza de trabajo que las jefas de hogar y las solteras sin hijos. ${ }^{30}$

En este trabajo analizamos las historias de vida y las percepciones de noventa mujeres urbanas, casadas y con hijos, que trabajaban en el momento de la entrevista (o habían trabajado alguna vez a lo largo de sus vidas). ${ }^{31}$ Se eligieron mujeres que

${ }^{28}$ Suárez analiza una cohorte de mujeres jóvenes (25 a 29 años), con datos de 1987. Muestra que $38.2 \%$ de ellas no había desempeñado una actividad laboral en los tres momentos seleccionados (antes del matrimonio, entre el matrimonio y el nacimiento del primer hijo, y en la fecha de la entrevista). No obstante, $20.4 \%$ sí había participado económicamente en esos tres momentos. En los casos restantes lo hicieron en una o dos de las etapas mencionadas.

${ }^{29}$ CRUZ PIÑEIRO, R. Volatilidad en el empleo femenino... Op. cit.

${ }^{30}$ SUÁREZ LÓPEZ, Leticia. Trayectorias laborales y reproductivas: una comparación entre México y España. Estudios Demográficos y Urbanos, vol. 7, ns 2-3, mayodiciembre 1992; CERRUTTI, M. Coping with Opposing Pressures... Op. cit.

${ }^{31}$ Los datos analizados fueron recolectados como parte de un proyecto de investigación sobre Fecundidad, Trabajo y Condición femenina llevado a cabo por Brígida García y Orlandina de Oliveira con el financiamiento de la Fundación 
Trayectorias laborares femeninas

desempeñaban actividades asalariadas y por cuenta propia, de tiempo completo y tiempo parcial, en ocupaciones manuales y no manuales, en tres ciudades mexicanas. ${ }^{32}$ Utilizamos en el presente trabajo una perspectiva de curso de vida para el análisis de las trayectorias y transiciones familiares y laborales. Nuestro propósito es ahondar en el estudio de la discontinuidad de las trayectorias laborales femeninas. Para ello caracterizamos las trayectorias en continuas y discontinuas, examinamos las diferencias entre sectores medios y populares, y argumentamos acerca de los diversos factores que ayudan a explicar las diferencias encontradas. ${ }^{33}$ Como se trata de un análisis de datos cualitativos obtenidos mediante entrevistas en profundidad en una muestra no probabilística, nuestros hallazgos no deben ser vistos como generalizaciones empíricas válidas más allá de los casos analizados. Ellos nos permiten más bien sugerir hipótesis que pueden orientar estudios futuros sobre el tema.

Los datos analizados incluyen a mujeres de 20 a 49 años, casadas y con hijos, que nacieron entre 1940 y 1970, años de implementación y consolidación del modelo de sustitución de importaciones en el país. Dada la amplitud de los tramos de edad de las entrevistadas, distinguimos dos cohortes de nacimiento, no con el propósito de analizar diferencias entre ellas, sino con la finalidad de descartar que las tendencias encontradas obedezcan a diferencias de edad entre las mujeres. La cohorte más antigua (mujeres nacidas entre1940 y 1955) ingresa por primera vez al mercado de trabajo, se

Rockefeller. Los principales resultados de esta investigación fueron publicados en García, B. y Oliveira, O. Trabajo femenino y vida familiar en México. Op. cit.

${ }^{32}$ Las entrevistas fueron realizadas en tres centros urbanos: Ciudad de México, principal área urbana del país; Mérida, localizada en el sureste, y Tijuana, una importante ciudad en la frontera con Estados Unidos. Se seleccionaron ciudades con características muy distintas para maximizar la heterogeneidad de los casos. No se perseguió el propósito de realizar una comparación entre ciudades.

${ }^{33}$ Se definió como pertenecientes a los sectores medios a mujeres con ocupaciones no manuales que contaban con algún grado de escolaridad superior a la secundaria. En los sectores populares se ubican aquellas con ocupaciones manuales y escolaridad menor que la preparatoria. 
casa y tiene hijos, durante un periodo de expansión de la economía mexicana, entre 1950 y 1970 aproximadamente. La segunda, en cambio (mujeres nacidas entre 1956 y 1970), si bien tuvo mayor acceso a las crecientes oportunidades educativas y a las políticas de control natal, ingresó al mercado de trabajo y emprendió la formación familiar en los años de agotamiento del modelo de sustitución de importaciones y de crisis económica (1970 a 1990), como tuvimos ocasión de ver. ${ }^{34}$ Estos procesos de crisis de los ochenta pueden haber afectado también las trayectorias laborales de la primera cohorte, sobre todo de las mujeres que ingresaron (o reingresaron) al mercado de trabajo en etapas avanzadas del curso de vida.

\section{Trayectorias continuas y discontinuas}

Las trayectorias laborales que describen las mujeres casadas y con hijos presentan una gran diversidad: unas son de larga duración, empiezan desde muy temprano durante la niñez y se prolongan hasta etapas avanzadas del curso de vida; otras, en cambio, son más cortas, se inician después del matrimonio o del nacimiento de los hijos; incluso en etapas más avanzadas de la vida. La mayor parte de las trayectorias se caracteriza por frecuentes entradas y salidas de la fuerza de trabajo.

Con la finalidad de caracterizar las trayectorias las diferenciamos por su grado de continuidad en continuas y discontinuas, evaluando la permanencia o salida de la actividad económica por un periodo de tiempo prolongado (más de 10 años, sin interrupciones), el que comprende diferentes momentos del ciclo familiar de las mujeres. De acuerdo con esta definición, las trayectorias laborales continuas se inician antes de la unión

\footnotetext{
${ }^{34}$ Las integrantes de la primera y segunda cohorte tenían en el momento de la entrevista (1990), entre 35 y 49, y 20 a 34 años, respectivamente. Como vimos en la primera parte de este trabajo, fueron precisamente las mujeres de 35 años y más, nacidas entre 1947 y 1952, las primeras en incrementar sus niveles de participación económica.
} 
conyugal y perduran después del casamiento o unión libre, y del nacimiento de los hijos. En contraste, las discontinuas pueden iniciarse antes o después del matrimonio, o en otro momento del curso de vida, presentando interrupciones de duración variable (de uno a varios años); y la permanencia en la actividad laboral se restringe sólo a uno o dos momentos del ciclo de vida: antes del matrimonio, antes de la maternidad, cuando los hijos no son chicos (12 años y más), después del divorcio o de la separación.

A partir del análisis de las interrelaciones entre las trayectorias educativas, laborales y familiares y de las percepciones verbalizadas por las mujeres entrevistadas, clasificamos los factores que propician la discontinuidad de las trayectorias en: personales, contextuales y familiares. Los motivos personales engloban enfermedades (propias o de familiares), migración (individual o familiar), accidentes de trabajo y/o retorno a la educación. La decisión de terminar los estudios (profesionales, técnicos o básicos) después del matrimonio y de la maternidad requiere en ocasiones el abandono de la actividad laboral por varios años, aunque muchas mujeres jóvenes trabajan y estudian simultáneamente. Los factores contextuales se refieren a aspectos vinculados con la dinámica de los mercados laborales $e$ incluyen despidos, cierre del lugar de trabajo, bajos salarios y condiciones insalubres de trabajo, entre otros. Las razones familiares son múltiples pero destacamos aquéllas vinculadas con los roles familiares a través de dos transiciones cruciales del curso de vida femenino: la unión conyugal, ya sea por casamiento o unión consensual, y el nacimiento de los hijos.

Los relatos de vida sugieren que las discontinuidades por casamiento difieren de las vinculadas con el nacimiento de los hijos: las primeras son de más larga duración (de 5 a 15 años de interrupción de la vida laboral), y denotan en forma más clara que las segundas la subordinación femenina ante el cónyuge. Las mujeres que abandonan la actividad laboral al casarse lo hacen con frecuencia por exigencia de los esposos, los que no les permiten salir de la casa a trabajar; pero también porque ellas comparten la idea que el mantenimiento económico del hogar es obligación del jefe 
varón. ${ }^{35}$ La interrupción de la vida laboral a causa del matrimonio responde a aspectos ideológicos de la construcción de género que establecen como "normal" y "natural" la división sexual del trabajo entre hombre proveedores y esposas-madres amas de casa. Un número importante de las mujeres que deja de trabajar al casarse sigue fuera del mercado de trabajo hasta que los hijos están grandes. El regreso a la vida laboral - si es que ocurre - se da en etapas avanzadas del ciclo familiar, si las necesidades económicas de la familia se vuelven apremiantes o si la unión conyugal se disuelve por separación o divorcio.

La discontinuidad de las trayectorias laborales por nacimiento o cuidado de los hijos es de más corta duración ( 1 a 3 años) y los aspectos ideológicos que la justifican asumen otros matices. ${ }^{36}$ En este

\footnotetext{
${ }^{35}$ Resulta interesante recoger la percepción que sobre el trabajo extra-doméstico tiene una parte importante de la población femenina mexicana, según lo refieren investigaciones de índole cualitativa. Así, por ejemplo, los estudios de Guttman en sectores populares mexicanos demuestran que todavía la gran mayoría de las mujeres entiende que cuando trabajan fuera de la casa para ganar un ingreso, están contribuyendo a ayudar al varón en su rol principal de proveedor. Existe complementariedad entre esta idea y la visión masculina de que el trabajo doméstico que pueden realizar eventualmente en el hogar es también una forma de ayuda a las tareas principales de las mujeres. GUTMANN, Mathew. Los hombres cambiantes, los machos impenitentes y las relaciones de género en México en los noventa. Estudios Sociológicos, vol. 9, n 33, 1993; ARIZA, Marina y OlivelRA, Orlandina de. Formación y dinámica familiar en México, Centroamérica y el Caribe. En FIGUEROA, Beatriz. (coord.) México diverso y desigual: aspectos sociodemográficos. México, El Colegio de México/Sociedad Mexicana de Demografía (Somede), 1999; GARCía, B. y OliveirA, O. Trabajo femenino y vida familiar en México. Op. cit.; CERRUTTI, M. Coping with Opposing Pressures... Op. cit.; OlIVEIRA, Orlandina de. La mujer en la actividad productiva: algunos comentarios. En GABAYET Luisa et alii. (comps.) Mujeres y Sociedad. Salarios, hogar y acción social en el occidente de México. México, El Colegio de Jalisco/Centro de Investigaciones y Estudios Superiores en Antropología Social (CIESAS), 1988; OliveIRA, Orlandina de. Familia y relaciones de género. En SCHMUKLER, Beatriz. (coord.) Familias y relaciones de género en transformación. México, Population Council/Edamex, 1998.

${ }^{36}$ En un análisis para una muestra nacional en Canadá también se encuentra que la discontinuidad en el empleo de las mujeres se relaciona en forma directa con las
} 
Trayectorias laborares femeninas

caso, cuando las mujeres contraen matrimonio pueden seguir trabajando en actividades extra-domésticas, pues en apariencia al "rol de esposa" se le concede un mayor grado de autonomía relativa que al de madre, en contraste con la situación anterior. Pero también el "rol de madre" expresa cierta flexibilidad en la medida en que la presencia de las mujeres en la crianza de los hijos es vista como esencial más que nada para los primeros años de vida, mientras se tolera que otras personas pueden hacerse cargo de los hijos. De ahí que varios sean los aspectos que llevan a la interrupción de la vida laboral de las mujeres cuando los hijos son chicos: falta de recursos económicos para pagar una empleada doméstica o enviar los hijos a guarderías privadas; ausencia de redes de apoyo; y presión de los cónyuges para que permanezcan en la casa en los años en que aquellos requieren más atención y cuidado. ${ }^{37}$ En situaciones extremas, las mujeres no retornan a la vida laboral por enfermedades prolongadas o incapacidad de los hijos, o debido a la creencia infundada de que las madres son las únicas capaces de cuidar adecuadamente los hijos hasta que alcancen la vida adulta.

\section{Diferencias entre sectores sociales}

Son conocidas las diferencias existentes en México entre sectores sociales en lo que respecta a niveles de escolaridad, edad a la unión, fecundidad y participación económica. Varias autoras han señalado además cómo las asimetrías de género se acentúan en los sectores con un acceso limitado a recursos sociales de diversa índole. $^{38}$ La comparación entre los relatos de vida de las

responsabilidades de crianza de los hijos. LI, P. S. y CURRIE, D. Gender Differences in Work Interruptions... Op. cit.

${ }^{37}$ García, B. y Oliveira, O. Trabajo femenino y vida familiar en México. Op. cit.; Cerrutti, M. Coping with Opposing Pressures... Op. cit.

${ }^{38}$ Educación, ingresos, entre otros, véase, DE BARBIERI, Teresita. Mujeres y vida cotidiana. México, Fondo de Cultura Económica (FCE)/Instituto de Investigaciones Sociales (IIS), UNAM, 1984; Quilodrán, 1990; GARCíA, B. y 
entrevistadas de sectores medios y populares urbanos muestra que las transiciones familiares las afectan en forma distinta en virtud del acceso diferencial a los recursos. ${ }^{39}$

En los sectores populares el casamiento tiene un papel más decisivo en la interrupción de la vida laboral que en las clases medias; aspecto que guarda relación con otros: el acceso a recursos socioeconómicos, la manera en que se concibe el matrimonio, y las relaciones de poder entre los cónyuges. Las mujeres que han enfrentado dificultades económicas durante la niñez y la adolescencia, que no han podido estudiar o tuvieron que dejar la escuela para trabajar, conciben casi siempre el matrimonio como una forma de obtención de apoyo moral y económico; y como una opción para dejar de trabajar y dedicarse a la crianza de los hijos. ${ }^{40}$ Otros estudios constatan a su vez una pauta de mayor sumisión y obediencia en las mujeres de mayor edad, menor escolaridad y escasos recursos económicos. En los sectores sociales más pobres, las mujeres no tienen asegurada su libertad de movimiento; se ven obligadas a pedir "permiso" al cónyuge para salir de la casa a trabajar, visitar los familiares o ir a un centro de salud. En México

OLIVEIRA, O. Trabajo femenino y vida familiar en México. Op. cit.; OLIVEIRA, Orlandina de. Experiencias matrimoniales en el México urbano: la importancia de la familia de origen. Estudios Sociológicos, vol. 13, no 38, 1995; ARIZA, M. y OLIVEIRA, O. Género y clase como ejes de inequidad... Op. cit.

${ }^{39}$ Moen señala, a partir de un análisis de las transiciones en la vida de mujeres de 35 a 59 años de edad en Estados Unidos, que el efecto diferencial de las transiciones y los roles familiares sobre la vida laboral depende, entre otras cosas, de los recursos y de la experiencia de las mujeres. La escolaridad y la edad contribuyen a que los cambios en los roles familiares afecten en forma diferencial la permanencia de las mujeres en la fuerza de trabajo. MoEN, Phyllis. Transitions in Mid-life: Women's Work and Family Roles in the 1970s. Journal of Marriage and the Family, vol. 53, febrero de 1991.

${ }^{40}$ García, B. y Oliveira, O. Trabajo femenino y vida familiar en México. Op. cit.; OlvieirA, O. Experiencias matrimoniales en el México urbano... Op. cit.; ARIZA, Marina. Migración, trabajo y género: la migración femenina en República Dominicana, una aproximación macro y micro social. Tesis de doctorado, México, El Colegio de México, 1997. 
Trayectorias laborares femeninas

este mecanismo de control es frecuente en los sectores campesinos y populares urbanos. ${ }^{41}$

En contraste, en los sectores medios las mujeres abandonan en menor medida la actividad extra-doméstica al casarse; pero el embarazo, el nacimiento y el cuidado de los hijos figuran como factores con un mayor peso en la interrupción de la vida laboral. Análisis estadísticos acerca de la importancia relativa de la presencia y la edad de los hijos en la propensión a trabajar en épocas de crisis económica indican efectivamente que los hijos pequeños (0-3 años de edad) constituyen un obstáculo para la participación económica de las mujeres de clase media; pero que en los sectores más necesitados se convierten más bien en un factor de estímulo de la participación. Las mujeres de estos sectores salen a trabajar aunque sea a escondidas del cónyuge para asegurar la manutención de sus hijos. $^{42}$

\section{Algunos factores explicativos de la permanencia o reingreso al trabajo}

El casamiento y el nacimiento de los hijos son transiciones familiares centrales en la explicación de la interrupción de las trayectorias laborales. Pero para entender la permanencia o el reingreso al mercado de trabajo es indispensable tener en cuenta otro conjunto de factores. En el análisis de los relatos de vida

${ }^{41}$ BeneríA, Lourdes y ROLDÁN, Marta. The Crossroads of Class and Gender. Industrial Homework, Subcontracting and Household Dynamics in Mexico City. Chicago, The University of Chicago Press, 1987; GARCíA, B. y OliveirA, O. Trabajo femenino y vida familiar en México. Op. cit.; Velasco ORTIZ, M. Laura. Comunidades transnacionales y conciencia étnica: indígenas migrantes en la frontera México-Estados Unidos. Tesis de doctorado, México, El Colegio de México, 1999.

42 Otros estudios dan elementos adicionales para fundamentar la posible existencia de una patrón diferencial de trayectorias laborales por sector social. Véase, BLANCO, Mercedes. Trayectorias laborales de una cohorte de mujeres de clase media en la Ciudad de México. Trabajo presentado en el XXI International Congress of Latin American Studies Association (LASA), LASA, Chicago, septiembre 1998; GARCíA, B. y OliveirA, O. Trabajo femenino y vida familiar en México. Op. cit. 
encontramos como relevantes los siguientes aspectos: los rasgos sociodemográficos de las mujeres, las estrategias de flexibilización de la actividad laboral, las redes de apoyo, los proyectos individuales y las necesidades económicas. En cuanto a los aspectos sociodemográficos, una fecundidad más reducida, mayores niveles de escolaridad, el desempeño de una carrera profesional o técnica (universitarias, maestras, enfermeras) y una mayor autonomía ante los cónyuges, con seguridad contribuyen a que las mujeres de clase media, en especial las más jóvenes, se inclinen más por una trayectoria laboral continua. ${ }^{43}$

Hay que considerar también que tanto las trayectorias laborales continuas como el reingreso a la fuerza de trabajo son posibles gracias a la puesta en marcha de diferentes estrategias de adaptación del trabajo a la vida familiar. La flexibilización de la actividad laboral que estas estrategias permiten adquiere diversas modalidades: disminución del tiempo dedicado al trabajo (paso del trabajo de tiempo completo al de tiempo parcial); adaptación de los horarios de trabajo al ritmo de la vida familiar (elección de turnos nocturnos, por ejemplo); cambio del trabajo asalariado con horario fijo a una actividad por cuenta propia de horario flexible. La venta de diferentes tipos de productos (ropas, joyas, cosméticos) o el establecimiento de un negocio familiar, constituyen mecanismos frecuentes de flexibilización del trabajo empleados tanto por las mujeres de los sectores medios como de los populares urbanos. ${ }^{44}$

Al igual que en otros países, la actividad económica de las mujeres mexicanas se caracteriza por el desempeño de trabajos más flexibles que los de los hombres en cuanto al horario, el lugar y la

\footnotetext{
${ }^{43}$ Pero las trayectorias laborales en los sectores populares son más intensas: las mujeres trabajan desde niñas en actividades más agotadoras, en jornadas más largas de trabajo y con una mayor carga de trabajo doméstico. Ellas no cuentan con empleadas domésticas, y los cónyuges participan menos en los quehaceres de la casa. GarcíA, B. y OliveIRA, O. Trabajo femenino y vida familiar en México. Op. cit.

${ }^{44}$ García, B. y Oliveira, O. Trabajo femenino y vida familiar en México. Op. cit.; Quilodrán, 1996, CERRUTTI, M. Coping with Opposing Pressures... Op. cit.
} 
Trayectorias laborares femeninas

posición en el trabajo. ${ }^{45}$ Con base en esto se argumenta en ocasiones que el trabajo por cuenta propia y el de tiempo parcial permiten a las mujeres mayor compatibilidad entre las actividades domésticas y extra-domésticas. Es necesario no minimizar el peso sobre la segregación en trabajos flexibles de factores socioculturales asociados a sus responsabilidades domésticas que justifican su exclusión de aquellos mejor pagados y con mayores posibilidades de ascenso. Cuando las mujeres al casarse y tener hijos cambian la naturaleza del trabajo (de tiempo completo a tiempo parcial, de asalariado a por cuenta propia) las consecuencias se manifiestan en un deterioro de la trayectoria laboral. Después de algunos o varios años de interrupción ellas regresan a actividades menos estables, peor pagadas y con menores posibilidades de movilidad social ascendente. $^{46}$

Por su parte, en los diferentes sectores sociales la existencia de redes de apoyo de familiares, amigos o vecinos hace más factible el desempeño en forma continua - o el reingreso - de actividades asalariadas de tiempo completo. En las clases medias la disponibilidad de recursos económicos para la contratación de empleadas domésticas o el envío de los hijos a guarderías privadas puede compensar la ausencia de las redes de apoyo. ${ }^{47}$

Es de interés subrayar asimismo que el significado que las mujeres atribuyen al trabajo extra-doméstico es fundamental para entender el mayor o menor grado de continuidad de la vida

\footnotetext{
${ }^{45}$ Como se señaló en la primera parte de este trabajo, la expansión de las actividades por cuenta propia y de los micronegocios ha contribuido a una creciente heterogeneidad de los mercados de trabajo en el país, aspecto que facilita la entrada de las mujeres en actividades más flexibles, pero también más precarias.

${ }^{46}$ Li y Currie señalan para el caso de Canadá que, a pesar de la sobrerepresentación en actividades de tiempo parcial y en posiciones desventajosas, las mujeres presentan una menor propensión que los varones a emplearse de tiempo completo, una vez que sus carreras han sido interrumpidas. LI, P. S. y CuRRIE, D. Gender Differences in Work Interruptions... Op. cit.

${ }^{47}$ García, B. y Oliveira, O. Trabajo femenino y vida familiar en México. Op. cit.; Oliveira, 1996.
} 
laboral. ${ }^{48}$ En efecto, cuando ellas asumen el trabajo extra-doméstico como parte de un proyecto de superación personal e independencia económica aumenta la propensión a participar con mayor continuidad en la actividad económica extra-doméstica. En este caso, las mujeres asignan a la vida laboral un significado igual o más importante que a la vida familiar, y conciben la ocupación elegida como una vocación. Lo anterior puede darse tanto en carreras profesionales y técnicas (trabajo social, enfermería, magisterio), propias de los sectores medios, como en las actividades manuales más frecuentes en los sectores populares (costura, bordado, culinaria)..$^{49}$

También los procesos económicos macro estructurales han dejado huella en las trayectorias analizadas. Como hemos mencionado, el contexto de recurrentes crisis económicas que ha caracterizado a la economía mexicana desde mediados de los ochenta ha determinado un incremento de las necesidades económicas familiares en los diferentes sectores sociales. Para compensar el bajo nivel salarial del jefe de hogar, las familias se han visto obligadas a recurrir al ingreso de varios de sus integrantes, en particular las mujeres. ${ }^{50}$ De ahí que seguramente muchas mujeres hayan permanecido o reingresado a la fuerza de trabajo en años recientes. Pero es pertinente tener en cuenta el carácter selectivo de

\footnotetext{
48 ID., IB.

${ }^{49}$ La distinción entre proyectos individuales y familiares la tomamos de García y Oliveira, quienes analizan el significado del trabajo en la vida de las mujeres de clase media y sectores populares y construyen una tipología a partir de un criterio de diferenciación básico: el compromiso subjetivo de las mujeres con la vida laboral. Véase también, CERRUTTI, M. Coping with Opposing Pressures... Op. cit.; GARCíA, B. y Oliveira, O. Trabajo femenino y vida familiar en México. Op. cit.

${ }^{50}$ González de la Rocha, Mercedes. The Resources of Poverty, Women and Survival in a Mexican City. Cambridge, Massachusetts, Blackwell, 1994; GARCíA, Brígida y PACHECO, Edith. Sectores sociales y participación económica familiar. Trabajo presentado en el XXI International Congress of Latin American Studies Asociation, LASA, Chicago, septiembre de 1998; RuBALCAVA, R. Necesidades, recursos y posibilidades... Op. cit.; OlIVEIRA, O. Políticas económicas, arreglos familiares y perceptores de ingresos. Op. cit.
} 
Trayectorias laborares femeninas

las consecuencias de los procesos macro estructurales sobre las familias y sus integrantes. ${ }^{51}$ En los sectores medios, la permanencia o el regreso al mercado de trabajo por necesidad económica hace parte con frecuencia de un proyecto familiar de mantenimiento del status social o de movilidad, mientras que en los sectores populares resulta de la necesidad de la supervivencia cotidiana y de asegurar la educación de los hijos. ${ }^{52}$

El análisis cualitativo nos ha permitido identificar un conjunto amplio de aspectos pertinentes en la caracterización y explicación del grado de continuidad o discontinuidad de las trayectorias laborales femeninas; a saber: las transiciones familiares (casamiento $y$ nacimiento de los hijos), los rasgos sociodemográficos de las mujeres (educación y fecundidad), las estrategias de adaptación del trabajo a la vida familiar, la existencia de redes de apoyo, los proyectos de desarrollo individual, y las necesidades económicas familiares. Para evaluar la importancia relativa de estos factores en distintos momentos del tiempo se requieren análisis estadísticos basados en información longitudinal de carácter probabilístico, no disponibles aún en México.

\section{Consideraciones finales}

El propósito de este trabajo ha sido doble. Señalamos, por un lado, las principales transformaciones estructurales (ocurridas en México de los años 30 a los 90) que permiten explicar la creciente participación económica femenina y los cambios en el perfil de las mujeres que trabajan. Analizamos, por el otro, las trayectorias laborales de un conjunto de mujeres casadas, y con hijos, cuyas vidas transcurrieron en ese periodo histórico. Inicialmente, destacamos el papel de la urbanización, la

${ }^{51}$ El aumento en la proporción de familias con dos o más perceptores de ingresos en el país entre 1984 y 1996, fue más acentuado en los casos en que el jefe varón ganaba menos de dos salarios mínimos mensuales. OliveIRA, O. Políticas económicas, arreglos familiares y perceptores de ingresos. Op. cit.

${ }^{52}$ García, B. y Oliveira, O. Trabajo femenino y vida familiar en México. Op. cit. 
industrialización y la terciarización como procesos de larga duración que han ampliado la estructura de oportunidades educacionales y de empleo disponibles para la población femenina. Vimos, asimismo, cómo la disminución de los niveles de fecundidad, resultado de la aplicación de las políticas de planificación familiar, y la reducción del número de años dedicados a la crianza de los hijos han hecho más factible la participación económica de las mujeres casadas en los mercados de trabajo de mediados de los setenta a los noventa.

La comparación de las vinculaciones entre familia y trabajo en dos periodos históricos, nos permitió mostrar la pérdida de importancia, en los años de crisis y reestructuración económica, del modelo familiar de jefe proveedor exclusivo característico de los años de sustitución de importaciones. La expansión de actividades más flexibles (trabajos por cuenta propia o de tiempo parcial), ha posibilitado una creciente participación económica de las mujeres, sobre todo de las casadas, a la vez que ha significado un deterioro de sus condiciones de trabajo. Las evidencias disponibles son contundentes: la mano de obra femenina participa más en la fuerza de trabajo en años recientes, pero lo hace en condiciones de más precariedad.

El análisis de la vida laboral de mujeres casadas y con hijos, nacidas entre 1940-1970, nos permitió caracterizar la heterogeneidad de sus trayectorias laborales, y sugerir de paso una serie de hipótesis sobre las diferencias entre sectores sociales y presentar algunas interpretaciones sobre los factores explicativos de la permanencia o reingreso de las mujeres al trabajo. Examinamos el papel de dos transiciones familiares centrales (casamiento y nacimiento de los hijos) en la discontinuidad de las trayectorias laborales. Señalamos asimismo, cómo las repercusiones de los cambios estructurales y de la ideología de género sobre la vida laboral asume un carácter selectivo y afecta en forma distinta a los sectores medios y a los populares urbanos. Las mujeres de los sectores populares urbanos, por su menor escolaridad y su mayor fecundidad, por la obediencia y sumisión a los cónyuges, así como por la creencia de que los 
varones tienen la obligación de mantener a la familia, interrumpen con mayor frecuencia su vida laboral al casarse que las mujeres de clase media. Estas últimas, por sus mayores niveles de escolaridad, menor fecundidad y concepciones distintas acerca de los roles familiares de esposa y madre tienden a interrumpir sus trayectorias laborales más por el nacimiento de los hijos. Las interrupciones que resultan del casamiento son mucho más prolongadas que aquéllas que se deben al nacimiento de los hijos.

Ilustramos de igual forma, las consecuencias negativas de la adaptación del trabajo a la vida familiar sobre las trayectorias descritas. El cambio en la naturaleza del trabajo extra-doméstico (de actividades asalariadas a por cuenta propia, del tiempo completo a tiempo parcial), como un mecanismo para armonizarlo con las responsabilidades domésticas, refuerza la segregación de las mujeres en actividades mal pagadas y con menores oportunidades de ascenso.

Destacamos, también la importancia de las redes de apoyo para el cuidado de los hijos y de los proyectos individuales de superación personal, como elementos explicativos de la permanencia o el reingreso de las mujeres casadas y con hijos a la actividad laboral. Señalamos por último, que la actividad económica femenina, como parte de proyectos familiares de movilidad social o supervivencia cotidiana, ha adquirido seguramente mayor importancia en el contexto actual de reiteradas crisis económicas. 